\title{
El estudio de las actitudes: Perspectivas psicológicas y sociológicas
}

José R. Torregrosa

\section{Planteamiento general}

El estudio de las actitudes es, hoy por hoy, una área de convergencia interdisciplinaria, dentro de las ciencias humanas. Numerosos estudios han sido llevados a cabo por investigadores en este terreno -psicólogos o sociólogos - utilizando como metodología desde la más controlada experimentación hasta el análisis cualitativo de textos y documentos, pasando por las encuestas de opinión y las entrevistas en profundidad. Esta variedad de contextos analíticos y metodológicos en que aparece el concepto de actitud, hace difícil la delimitación de su alcance teórico-oonceptual, de su papel dentro de las hipótesis de que suele formar parte. Ahora bien, aunque el estudio de las actitudes pretendió conectar la teoría de la cultura -0 más específicamente, la sociología - con la psicología individual, desdo el principio, sin embargo, este estudio fue llevado a cabo con fines esencialmente psicológicos (1). Esta tendencia apuntada por Znaniecki, refiriéndose a la monumental obra que escribió con Thomas (2), puede decirse que persiste todavía. Y persiste, en gran medida, porque, como les ocurrió a Thomas y Znaniecki, todavía se conceptualizan las actitudes como "tendencias subjetivas a actuar" (3), como características individuales.

En esto sentido, la tesis fundamental de este trabajo es que para un estudio adecuado de las actitudes es indispensable un marco conceptual y un nivel analítico que tengan en cuenta de manera sistemática las incontrovertibles realidades de la estructura sociocultural.

Tal afirmación es, conscientemente, excesivamente genérica y parece, en principio, obvia. Sin embargo, a pesar de que, en principio, muy pocos estudiosos estarían en desacuerdo con ella, el hecho es que en la mayoría de los estudios empiricos sobre actitudes y opiniones, e incluso, en los phanteamientos teórioos más recientes, el contexto sociocultural queda como

(1) Znanieckr, F.: Cultural Sciences. University of Illinois Press. Urbana, 1952. Pág. 237.

(2) Thomas, W. I. y Znaniecki, F.: The Polish Peasant in Europe and America. Alfred A. Knopf, Nueva York, 1927. Para extractos fundamentales de esta obra véase: Thomas, W. I.: On Social Organization and Social Personality. Janowitz, M (Ed.). "The University of Chicago Press", Chicago, 1966.

(3) ZnANieckı, F.: Op cit, pág. 238. 
un trasfondo difuso, como algo dado, cuyo análisis es considerado como periférico. En mi opinión, sin embargo, el análisis de la estructura y dinámica social y cultural, en sus distintos niveles do especificidad y en sus distintos subsistemas institucionales, resulta un marco de referencia indispensable y central en el estudio de las actitudes.

En este sentido, una teoría sobre las actitudes no sólo debe ser psicológica, es decir, tener como marco conceptual de referencia los elementos componentes de la estructura y dinámica de la personalidad, sino incluir en sus proposiciones conceptos referentes a la estructura social y sus procesos. Porque existen actitudes cuya naturaleza y significación no se deben tanto al hecho de que sean elementos intrapsíquicos de personas en particular, sino más bien a que constituyen características esenciales de situaciones en las que, el mero hecho de estar en ellas, independientemente de la personalidad específica de los actores, determina su adopción (4). El lacus de las actitudes en este caso no es la personalidad, sino el modo en que la situación está estructurada, es decir, la organización social y los procesos de interacción que acontecen en su seno. Los modos especiales de interrelación e interdependencia de los elementos componentes de la estructura sociocultural -en sus diferentes subsistemas - determinan a su vez modos de adaptación interpersonal e intergrupal cuya naturaleza, dialécticamente, proyecta no sólo los respectivos status y roles de los actores, sino también sus respectivos comportamientos, experiencias y orientaciones.

Esto no significa, en ningún momento, infravalorar el papel que en el estudio de las actitudes, o de cualquier otra realidad social, desempeña la teoría de la personalidad (5), sino más bien pretende poner de manifiesto la convioción de que sin una introduoción sistemática y explícita de conceptos sociológicos en la teoría e investigación empírica sobre actitudes, nuestras posibilidades de comprensión quedan sustancialmente limitadas y, en muchos casos, encerradas en un tipo de pensamiento caracterizado por un claro reduccionismo psicologista.

Ciertamente que los objetivos específicos de una investigación determinada establecen de suyo el marco teórico conceptual de referencia. Pero incluso en estudios cuyos propósitos sean estrictamente psicológicos -por

(4) Becker, H. S. y Geer, B.: "The Fate of Idealism in Medical School". American Sociological Reviezw, febrero, 1958. Págs. 50-56; L. Arangures, J. L.: "Comportamientos políticos reales y verbales en la circunstancia española". Cuadernos para el Diálogo, junio y julio, 1967.

(5) Coincido con la posición de Inkeles de que una teoría de la personalidad es necesaria para la teoría sociológica. Véase Inkeles, A.: "Personality and Social Structure", en Merton, Broom y Cottrell (Eds.). Sociology Today, 1959; Harper Torchbooks. Nueva York, 1955. Vol. II, págs. 249-276. 
ejemplo, ¿cómo se aprende cualquier actitud? (6)- es necesario hacer entrar nociones y elementos conceptuales que rebasen el ámbito del estudio de la psicología. No basta, en mi opinión, decir que tales o cuales situaciones u objetos son "estímulos sociales" a los que el sujeto reacciona o evalúa de algún modo. Las condiciones específicas en que operan tales situaciones, su estructura y sus procesos, y la posición que el sujeto ocupa dentro de las mismas, deben ser analizados. El análisis del campo sociocultural en el que un individuo puede tener tal o cual actitud es tan fundamental para una explicación de la misma -o del comportamiento que esperamos determine - como las funciones que tal actitud puede desempeñar para el individuo mismo.

Con lo anterior, pues, quiero poner de manifiesto que muchas actitudes no son sólo sociales en el sentido de que su objeto es un valor social cuya contrapartida subjetiva son las actitudes, o que éstas están socialmente determinadas - son aprendidas en los procesos de interacción social-, sino también en el sentido de que constituyen propiedades o características de grupos y situaciones sociales, creencias y modos de evaluación de los mismos, independientemente de que lo sean de los miembros individuales de tales grupos y situaciones; y que, por lo tanto, la perspectiva teórica adecuada para su comprensión y explicación debe ser una perspectiva sociológica. Los grupos, aunque menos visibles que los individuos, son tan reales como las personas en el contexto de la estructura social global (7). Y en cuanto tales, tienen determinadas formas de organización, determinados intereses, determinadas perspectivas, determinadas formas de ver y evaluar la realidad y determinados modos de actuar sobre la misma. Son, en definitiva, sistemas de acción social cuyos procesos internos y recíprocos configuran situaciones históricas específicas a cuya estructura habría que referir la naturaleza y funciones de muchas actitudes sociales, y no sólo correlacionarlas con ciertos rasgos, más o menos profundos, de la personalidad, u observar y describir su distribución estadística en una población dada.

\section{Algunas perspectivas teóricas}

\section{La teoria de la disonancia cognoscitiva de Festinger.}

Una de las orientaciones teóricas que ha originado, quizás, mayor número de investigaciones experimentales sobre el cambio de actitudes, es la

(6) Sherif, M. y Cantril, H.: The Psychology of Ego-Involvemientes. Wiley, Nueva York, $194 \%$.

(7) Warriner, Ch. K.: "Groups Are Real: A Reaffirmation, en Stoodley, B. H. (Ed.), Society and Self. The Free Press, Nueva York, 1962. Págs. 29-38. 
teoría de la disonancia cognoscitiva de Festinger (8). No nos interesa aqui evaluar tal teoría ni los resultados de los experimentos en que se han ido poniendo a prueba sus derivaciones (9). Tal labor, en realidad, sólo puede hacerse en el contexto de la investigación empírica misma. Lo que sí nos interesa, en cambio, es señalar algunos aspectos generales de su perspectiva en relación con la tesis que hemos apuntado más arriba.

Las dos hipótesis básicas de Festinger son:

"1. La existencia de disonancia en una persona, puesto que es psicológicamente incómoda, le motivará a que intente reducir la disonancia y llegue a la consonancia.

2. Cuando existe disonancia en una persona, ésta, además de intentar reducirla, evitará activamente situaciones e información que probablemente incrementarían la disonancia" (10). La disonancia es la existencia de relaciones cognoscitivas -creencias, opiniones o actitudes-- que no cuadran entre sí. "Dos elementos eśtán en una relación disonante si, considerados ellos solos, lo contrario de uno de los elementos se siguiese del otro... X e Y son disonantes si no - X se sigue de $\mathrm{Y}^{\prime \prime}$ (11). Así, pues, existe en las personas "una presión que produce relaciones consonantes entre las cogniciones y que tiende a evitar y reducir la disonancia" (12). La disonancia cognoscitiva "puede ser considerada como una condición antecedente que conduce a la actividad orientada hacia la reducción de la disonancia del mismo modo que el hambre conduce a actividad orientada hacia su reducción" (13).

Pretender que estas breves citas puedan dar una visión exacta de la fecundidad de las formulaciones de Festinger sería una enorme injusticia para uno de los hombres a quienes más debe la Psicología Social actual. Ahora bien, sí creo que son indicativas de algunas de las limitaciones que señalábamos más arriba en los estudios sobre actitudes. En primer lugar, la teoría de la disonancia cognoscitiva hace referencia, fundamentalmente, a la estructura y procesos intrapsíquicos, cuya explicación y predioción se hace, a su vez, postulando un principio psicológico. La estructura de las actitudes se mantiene porque en el individuo existe una tendencia hacia la consistencia, y cambian porque la inconsistencia entre ellas le motivan a su reorganización. El indiividuo, y en especial una tendencia de su estructura cognoscitiva, es la instancia explicativa última. Ahora bien, ni esa tendencia puede ser con-

(8) Festinger, L.: A Theory of Cognitive Dissonance. "Stanford Univ. Press". Stanford, 1957.

(9) Brehem, J. W. y Cohen, A. R.: Explorations in Cognitive Dissonance, Wiley, Nueva York, 1962.

(10) Festinger, L.: Op. cit., pág. 3.

(11) Festinger, L.: Op. cit., pág. 13.

(12) Festinger, L.: Op. cit., pág. 9.

(13) Festinger, L.: Op. cit., pág. 3. 
siderada como universal, ni es igualmente relevante en todas las situaciones. El hombre y los grupos pueden, sencillamente, dejar de establecer relaciones entre sus conocimientos, actitudes o creencias, encapsulándolas en compartimentos estancos de modo que su comunicación sea mínima. Cientamente que, en este caso, la teoría de la disonancia cognoscitiva nos diría que se trata de relaciones "irrelevantes" entre elementos cognoscitivos, sobre los que tiene poco que decir. Pero en muchos casos esa "irrelevancia" es demasiado significativa desde el punto de vista de otros procesos psicosociológicos para que no merezcan un análisis más detenido. Como afirman Katz y Stotland, "el modelo lógico del hombre es demasiado sencillo para tener en cuenta toda la complejidad del comportamiento social a pesar de la persuasiva reedición que de él han hecho los fenomenólogos" (14).

En segundo lugar, la teoría de la disonancia cognoscitiva no analiza con suficiente atención la estructura de las situaciones en que se produce o no la disonancia, y si se produce, las condiciones que conducen a uno u otro modo de reducirla. Esta especificación de condiciones, en mi opinión, a lo que debería llevar es a un análisis más completo de las variables sociológicas que tipifican la estructura de determinadas situaciones. Teorizar sobre la estructura y dinámica de las actitudes sin tener en cuenta de manera sistemática este tipo de variables es, en mi opinión, sólo una parte del problema. Citando de nuevo a Katz y Stotland, "una Psicología Social adecuada debe tener en cuenta el mundo exterior a la persona, y no en la forma en que lo hacen los "teóricos del campo", quienes se interesan sólo por los factores ambientales tal y como aparecen representados en el "campo vital" del individuo. Del mismo modo que el conocimiento del estímulo es importante para el psicólogo de laboratorio que estudia procesos sensoriales, el psicólogo social debe considerar el ambiente social independientemente de como sea percibido por el sujeto" (15).

El mismo tipo de consideraciones podríamos hacer con respecto a otras concepciones sobre la organización y cambio de las actitudes, basadas fundamentalmente en el principio de la consistencia, como el principio de la congruencia (congruity) de Osgood y Tannenbaum (16) o el del equilibrio (balance) de Heider (17), a quien realmente se debe el haber extendido, desde

(14) Katz, D. y Stotland, E.: "A Preliminary Statement of a Theory of Attitude Structure and Change" en Koch, S. (Ed.) Psychology: A Study of a Science. Mc. Graw Hill, Nueva York. 1959. Pág. 447.

(15) Katz y Stotland: Op. cit.

(16) Osgood, C. E. y Tannenbaum, P. H.: "The Principle of Congruity in the Prediction of Attitude Change". Psych. Bull. Vol. 62, 1955, págs. 42-45.

(17) Hexder, F.: "Attitudes and Cognitive Organization". Journ of Psych. Vol. 21, 1946, págs. 107-112. 
los supuestos de la Psicología de la Gestalt, la noción de consistencia a la teoría de las actitudes.

Hemos considerado la teoría de Festinger por ser la de un alcance más general y por haber originado, sistemáticamente, un gran número de investigaciones experimentales (18). En definitiva, y como afirma Zanjonc, "el concepto de consistencia subraya y presume la racionalidad humana. Sostiene que el comportamiento y las actitudes no son sólo consistentes para el observador objetivo, sino que los individuos mismos intentan verse a sí mismos como consistentes. Supone que la inconsistencia es un estado incómodo que moviliza presiones para eliminarlo o reducirlo. Pero en los modos por los que se consigue la consistencia en las actitudes y el comportamiento humanos podemos observar una sorprendente falta de racionalidad" (19').

\section{Katz y la perspectiva funcional.}

Este énfasis en el aspecto cognoscitivo de las actitudes y en el principio de la consistencia del enfoque a que hemos hecho referencia, tiene su contrapartida en el enfoque funcional o motivacicnal. Esta perspectiva sitúa el estudio de las actitudes no sólo en referencia a la dimensión cognoscitiva de la personalidad, sino a la estructura y dinámica de la personalidad en general, y, especialmente, a lo que se considera como el núcleo de la misma: su sistema motivacional. "Las opiniones y actitudes, como todo el comportamiento, constituyen al mismo tiempo parte de los datos desde los que se infiere la personalidad y están en función de la misma. Explicamos la consistencia en las opiniones de una persona en los mismos términos teóricos que utilizamos para explicar sus consistencias en los gestos, la expresión emocional o sus acciones motivadas" (20).

Un claro antecedente de esta perspectiva lo constituyen las investigaciones sobre "La personalidad autoritaria" (21), aunque los investigadores de Berkeley utilizaron un marco conceptual psicoanalítico más definido.

Pero la formulación más sistemática y reciente de este enfoque es la de Katz (22). La posición de este autor no desconoce la operatividad del prin-

(18) Para un buen resumen sobre esta temática véase: Cohen, A. R.: Attitude Change and Social Influence. Basic Books, Nueva York, 1964.

(19) Zajonc, R. B.: "The Concepts of Balance, Congruity, and Dissonance", en Steiner y Fishbein (Eds.) Current Studies in Social Psychology. Holt, Rinehardt and Winston, Nueva York, 1965. Pág. 27.

(20) SMITH, M. B.; BRUNer, J. S., y Whrre, R. W.: Opinions and Personality. 1956, Wiley, Science Ed., Nueva York, 1964. Pág, 29.

(21) Adorno, T. W.; Frenkel-Brunswick, E.; Levinson, D. J. y Sanford, R. N.: The Authoritarian Personality. Harper, Nueva York, 1950. Traducción castellana; Ed. Proyección, Buenos Aires, 1965.

(22) KaTz, D.: "The Functional Approach to the Study of Attitudes", en Hollander, E. P. y Hunt, R. G. (Eds.). Current Perspectives in Social Psychology. Oxford University Press Nueva York, 1963 Págs. 240-250; Katz y Stotlavd: Op. cit. 
cipio de la consistencia en la organización y dinámica de las actitudes, pero hace notar que es más limitada de lo que los autores de los modelos cognoscitivos parecen suponer. Específicamente, el principio de la consistencia debería tenerse en cuenta, sobre todo, en las relaciones entre los distintos componentes de una misma actitud, más bien que entre distintas actitudes del individuo. "La tendencia hacia la consistencia se produce más fuertemente dentro de los límites de una misma actitud; dentro de la misma hace que los componentes de la actitud sean congruentes entre sí. Las inconsistencias entre distintas actitudes pueden producirse más fácilmente que entre los componentes de una misma actitud. La razón do esto es que los componentes cognoscitivo, afectivo y activo están vinculados al mismo objeto, representando así una unidad molar del funcionamiento psicológico" (23).

En este sentido, por ejemplo, están orientados los experimentos de Rosenberg (24). Ahora bien, el énfasis de este enfoque radica en su esfuerzo por establecer relaciones entre las actitudes y los diferentes motivos del individuo. "El supuesto básico de esta orientación -escribe Katz- es que tanto la formación como el cambio de las actitudes debe ser entendido en términos de las necesidades a las que sirven $\mathrm{y}$, dado que estos procesos motivacionales son distintos, de igual modo serán distintas las condiciones y formas del cambio de actitudes" (25). En este sentido, las preguntas fundamentales serían: ¿De qué le sirve a un individuo tener o expresar tales o cuales actitudes? ¿Qué función desempeñan las actitudes en la economía psicológica total de una persona? Según Katz, estas funciones son básicamente cuatro:

a) La función instrumental, utilitaria o adaptativa. Esta función hace referencia a que los individuos intentan maximizar las recompensas y minimizar los castigos, desarrollando actitudes favorables hacia aquellos objetos y personas que se asocian con la satisfacción de sus necesidades, y actitudes desfavorables para aquellos objetos y personas que se asocian con la frustración de esas necesidades.

b) La función defensiva la desempeñan aquellas actitudes que tienden a proteger en el individuo cierta imagen sobre sí mismo o sobre la realidad exterior que se ve amenazada por hechos que no concuerdan con la misma. Estas actitudes tienen su origen en conflictos e inseguridades internos y su

(23) Katz y Stotland: Op. cit., págs. 444.

(24) Rosenterg, M. J.: An Analysis of Affective-Cognitive Consistency, en Rosenberg, Hovland, Mc. Guire, Abelson y Brehm: Attitude Organization and Change. Yale University Press, New Haven, 1960. Págs. 15-64.

(25) KaTz: Op. cit., pág. 342. 
expresión proporciona como una salida a las tensiones generadas por los mismos. A corto plazo, las actitudes defensivas pueden ser instrumentales, puesto que ofrecen una posibilidad de descargar la tensión; pero a largo plazo pueden perturbar la adaptación normal del individuo.

c) La función expresiva o comunicativa (value-expresive) hace referencia a que el individuo no sólo siente la necesidad de "defenderse" o maximizar las recompensas del entorno, sino también de manifestar sus propios valores o creencias básicas, aquellos que están más ligados a su propia identidad personal. Al expresar estos valores el individuo anuncia a los demás la imagen que tiene de sí mismo o que quiere alcanzar. Verifica y delimita, de este modo, su propia identidad personal, o alguna de sus "sub-identidades".

d) La función cognoscitiva. Esta función la cumplen aquellas actitudes que proporcionan al individuo una visión más o menos organizada de su mundo. El hombre necesita dar un sentido a su experiencia, encajar dentro de un maroo de referencia significativo aquello que ocurre en torno suyo. Esta función pueden cumplirla tanto la aceptación de ciertos estereotipos como una auténtica inquietud por el saber científico. Psicológicamente, la función es similar: ordenar "con sentido" los datos de la experiencia.

No cabe duda de que el enfoque funcional es, teóricamente, más completo y sugestivo que los modelos cognoscitivos. Ofrece un esquema interpretativo más amplio, en el que son debidamente sopesadas las complejidades de la estructura y del funcionamiento de la personalidad, de su papel como determinante de las actitudes. La consecuencia de este enfoque, desde el punto de vista de la estrategia para el cambio de actitudes, sería que lo fundamental no es tanto proporcionar nueva información sobre el objeto sino modificar las bases motivacionales sobre las que se configura la actitud misma. Ahora bien, el marco de referencia del enfoque funcional sigue siendo psicológico, haciendo hincapié, sobre todo, en estructuras y procesos intrapsíquicos. La enumeración de motivos posibles con los que pueden estar vinculadas las actitudes, nos revela de por sí un cierto eclecticismo teórico psicológico: teoría conductista del aprendizaje, psicoanálisis, psicologías del yo y la psicología de la gestalt. Por el contrario, no se hace referencia a los procesos de interacción social ni a situaciones específicas en las que cristalizan determinadas actitudes y mativos, $y$ en referencia a los cuales tanto las actitudes de individuos concretos como las de grupos encontrarían una explicación más completa, más socio-psicológica (26). Porque, a pesar

(26) WRight Milus, C.: "Situated Actions and Vocabulaires of Motives", en Wright Mills: Power, Politics and People. Ballantine Books, Nueva York, sin fecha. Traducción castellana. Ed. Paidós. 
de que Katz y Stotland, como indicábamos anteriormente, hacen notar la necesidad de un conocimiento de la situación para una especificación de las condiciones en que se producen o cambian las actitudes, una teoría de las situaciones - es decir, una perspectiva socio-cultural- no está integrada o articulada en su enfoque funcional. Pero este conocimiento, habría que aclarar, no sólo es importante porque la situación proporciona al individuo el "estímulo", sino porque individuo y situación son indesligables empíricamente. Como afirma Parsons, "los dos tipos de sistemas (la personalidad y el sistema social) no sólo son interdependientes, sino que se interpenetran" (27). En este sentido, y en la medida en que el estudio de las actitudes es un modo de estudiar esas interrelaciones, toda teoria sobre las actitudes debe contener proposiciones que puedan ser referidas tanto a la personalidad como al sistema social.

\section{La aportación de Kelman.}

Desde esta orientación, un paso hacia adelante lo representa el tipo de formulación de Kelman (28). El esfuerzo de Kelman se enoamina a superar la dicotomía entre conformismo público y aceptación privada. Esta dicotomía sigue manteniendo el supuesto de que, por una parte, está la esfera interna del individuo $\mathrm{y}$, por otra, su esfera pública, su fachada, sin solución de continuidad. Los "lavados de cerebro", sin embargo, pusieron de manifiesto que es posible sostener creencias y actitudes cuya razón de ser no radica ni en un mero conformismo instrumental -estas actitudes y creencias no desaparecen tan pronto como desaparece su función instrumental- ni en una plena integración con el resto de las actitudes y creencias del individuo. Estas actitudes y creencias parecen "estar aisladas del resto de los valores de la persona y ser básicamente dependientes del apoyo externo" (29). Es decir, que son características fundamentales de la estructura misma de la situación. De esta constatación Kelman llega a una formulación del cambio de las actitudes en que el punto de referencia ya no es tanto el individuo como los procesos mismos de interacción social. En la tipificación de sus tres procesos de influencia social - sumisión, identificación e interiorización - no sólo especifica las bases motivacionales en el individuo para el cambio, sino también la fuente del poder del agente de influencia (influecing agent) y el modo en que éste consigue la prepotencia

(27) Parsons, T.: "Psychology and Sociology", en Gillin., J. (Ed.). For a Science of Social Man. Mc. Millan, Nueva York, 1945. Pág. 71.

(28) Kelmax, H. C.: "Processes of Opinion Change". Public Opinion Quarterly 1961. Vol. 21. Págs. 57-58.

(29) Kelman: Op. cit., pág. 58. 
de la respuesta inducida. La introducción sistemática de variables como el poder del agente de influencia, el modo en que éste induce al actor a aceptar la influencia, confiere al modelo de Kelman un nivel analítico propiamente psico-sociológico. Las hipótesis que de él pueden derivarse no sólo se refieren al actor o sus características, sino también a alter, bien sea un individuo o un grupo, a sus interrelaciones y a la situación en que se producen.

\section{Conclusión}

¿Cuáles son las implicaciones de la anterior discusión? Una, en mi opinión, resulta en principio clara: que en el estudio de las actitudes al nivel de análisis psicológico no es suficiente. Requiere una perspectiva psico-sociológica en que los concoptos y variables sociológicos sean considerados con todas sus consecuencias teóricas y empíricas. Mi insistencia sobre este punto se debe a que el peligro de "psicologismo" es algo constantemente presente en nuestros modos de conceptualización y en nuestros instrumentos de investigación. El individuo resulta siempre más visible, más inmediato, más accesible. Sus contornos físicos están perfectamente delimitados; es lo que y a quien normalmente vemos. Por lo tanto, la tendencia a erigirlo como origen y causa última de acciones, creencias, actitudes, etc., se ve reforzada por nuestros propios mecanismos perceptivos (30). De aquí a comenzar a comparar individuos en algo que, supuestamente, está en ellos más bien que en las situaciones totales de las que forman parte no hay más que un paso. La prevalencia del concepto de actitud como variable psicológica latente es un buen ejemplo de ello. Refiriéndose a la falta de correspondencia entre actitudes verbales y comportamiento, De Fleur y Westie escriben: "Existen claramente factores situacionales tales como normas del grupo, roles, definiciones de la situación, y otras presiones sociales que materialmente mediatizan las respuestas en situaciones que implican o comportamiento verbal o acción manifiesta. Tales presiones sociales "mediatizadoras" parecen explicar mejor la falta de correspondencia que la noción de actitudes reales concebidas como variables latentes" (31). Ahora bien, si muchas actitudes y creencias no son tanto características específicas individuales, sino elementos constitutivos de la estructura de determinadas situaciones o grupos, ¿no las explicaríamos

(30) Heider, F.: "Social Perception and Phenomenal Causality", en Tagiuri, R. y Petrullo, L. (Eds.): Person Perception and Interpersonal Behavior. Stanford Univ. Press. Stanford, 1958. Págs. 1-21.

(31) De Fleur, M. L. y Westie, F. R.: "Attitude as a Scientific Concept". Social Forces, vol. 42 , págs. 26 . 
más plenamente si las refiriésemos a las situaciones y a los grupos mismos en vez de al funcionamiento y características psicológicas de los individuos? El hecho de que determinadas actitudes o creencias sean expresadas por ciertos grupos o instituciones, ¿qué funciones tiene para ellos mismos o para la sociedad en general? Que determinadas clases saciales sean aparentemente más "autoritarias" que otras, ¿qué función tiene para esas mismas clases, para el contexto más amplio de la sociedad global, o para un grupo dominante? ¿Son "sus" actitudes y creencias? ¿O reflejan situaciones cuya naturaleza --incluidas las vivencias de quienes en ellas se encuentran- sólo adquiere sentido dentro de un proceso histórico-dialéctico más amplio?

Yo no creo que estas preguntas - hechas en términos muy genéricos, pero que cabría hacérselas sobre muchos estudios- sean empiricamente irrelevantes. Todo depende de que sepamos trascender una determinada "lógica reconstruida" (32), considerada como el único método científico y de que contemplamos con cautela el "individualismo" o "psicologismo" de muchos de nuestros métodos, para que lo que es, en principio, un "individualismo metodológico" no sea también un individualismo conceptual y explicativo, falto de aquello que, en última instancia, confiere significación a todo comportamiento humano: su dimensión histórica.

(32) Kaplan, A.: The Conduct of Inquiry: Methodology for Behavioral Science. Chandler Publishing Comp., San Francisco, California, 1964. 